\title{
Bridging Tao Psychotherapy and Person-Centered Psychotherapy: One Psychotherapist's Journey
}

\author{
Eunsun Joo1)
}

\begin{abstract}
This study aims to seek "What are the similarities and differences of Tao psychotherapy and person-centered psychotherapy“ by using biographical narrative approach. The subject summarizes the results, called the psychotherapist who is a native Korean with Western training in Person-centered psychotherapy, exposed to Tao psychotherapy returning to her homeland. Her expertise in both Tao psychotherapy and person-centered experience shows that the similarities are two points that both approaches seek therapist's maturing his/her personality, and therapist's presence itself can be therapeutic. In terms of differences, issues related to dealing "self-in-relations", holistic approach and format of therapy being brief and directive are presented. Finally, discussion will be suggested in the context of psychotherapeutic culture and national culture.
\end{abstract}

Keywords: Tao Psychotherapy, Person-centered Psychotherapy, Biographical Narrative Approach, Psychotherapeutic Culture

\section{Introduction}

Psychotherapy needs to be understood in the context of culture. It can be described that Western culture is democratic, egalitarian and pragmatic whereas Korean culture can be explained as patriarchy, hierarchy and keeping face (Chae-myun) is valued. At the same time psychotherapy has its own culture of helping others and the importance of psychotherapist training. This article seeks to set a stage for dialog regarding how one can bridge a major Western psychotherapeutic approach and Korean cultural based psychotherapeutic approach. In this context, the researcher will use biographical narrative method comparing Person-centered psychotherapy and Tao psychotherapy. Biographical narrative method which is one of qualitative research approach concerned with the reconstruction of life histories and the composition of meaning based on biographical narratives and documents[1][2]. The research question of this study stems from a natural curiosity developed by a psychotherapist who was

Received(October 21, 2019), Review Result(1st: November 13, 2019, 2nd: December 18, 2019), Accepted(February 20, 2020)

1) (Professor) 01369 Dept. Psychology, Duksung Univ., 33, Samyang-ro 144-gil, Dobong-gu, Seoul, Republic of Korea

email: esjoo77@gmail.com 
struck by how different Korean clients were compared to those in America. Korean clients, coming from such a different cultural background, lack knowledge of psychotherapy and have entirely different expectations about what any kind of helping relationship should involve. Indeed, helping professionals educated in Western Therapeutic traditions almost universally report experience difficulties when trying to apply Western approaches based on individualism and egalitarianism to Korean individuals seeking psychological assistance (Joo, 2006)[3]. As a Korean born psychotherapist, trained in the West, returned to Korea to practice was introduced to Tao psychotherapy. Tao psychotherapy was developed by Dr. Rhee Dongshick, the founder of Korean Academy of Psychotherapists (KAP), to professionals seeking to overcome the limitations of Western psychotherapy with Koreans. Tao psychotherapy can be explained as a synthesis of Eastern and Western psychotherapies. It seeks to integrate psychoanalytic, existential, humanistic, and transpersonal, and Eastern perspectives in a single coherent approach (Craig, 2007)[4]. As a native Korean with Western training in Person-Centered psychotherapy, the researcher soon began to wonder, "What are the similarities and differences of Tao Psychotherapy and Person-centered Psychotherapy?" This paper is a beginning attempt to address this question.

Person-centered therapy was developed by Carl Rogers which uses a non-authoritative approach that allows clients to take more charge in counseling session so that, in the process, clients are able to solve their own issues. The therapist listens as a empathic facilitator without judgment and the therapist is there to encourage and support the client by providing therapeutic conditions in therapy such as genuineness, unconditional positive regard and empathic understanding. Tao psychotherapy (Rhee, 1993, 1995)[5][6], provides a fundamentally humanistic approach to depth psychology and psychotherapy. Tao psychotherapy primarily offers a philosophical (i.e., ethical, epistemological, and ontological) base for psychotherapy, and draws its philosophical foundations from ancient Eastern thought and practice. The goals of therapy of person-centered and Tao therapy are both similar. Ultimately, the therapist needs to provide necessary and sufficient therapeutic conditions for a client's self-actualization. In one of his distinguished books, "On Becoming a Person (1961)[7]", Rogers shares his philosophy with the master of Taoism, Lao-tzu :

As the passage of time has enabled me to look more objectively at what I said, I feel satisfaction on two counts. Firstly, my confidence in the human organism, when it is functioning freely; and secondly, the existential quality of satisfying living, a theme presented by some of our most modern philosophers. According to Lao-tzu, more than twenty-five centuries ago, he beautifully expressed, "The way to do is to be." 
Furthermore, according to Craig (2007)[4], Carl Rogers carried a hand written copy of the whole of Bynner's (1944)[8] translation of this chapter 17 in his wallet wherever he went. Rogers did not hesitate later in his life, to testify of his appreciation of the Tao Te Ching (see Rogers, 1961, p.164; 1973, pp.12-13)[7]. The sage is self-effacing and scanty of words. When his task is accomplished and things have been completed, all the people say, "We ourselves have achieved it!" (Chapter 17, Wu, 1961)[9].

In order to understand Tao psychotherapy and person-centered psychotherapy, one needs to understand wu-wei. In the Confucian Analects, it is said that the King Shuen ruled his country with wu-wei. "Wu-wei is sometimes translated as 'non-doing' and is a central tenet of the Tao-teaching. It means designating nonintervention in the natural course of things, spontaneous action devoid of premeditation and deliberate intention yet wholly appropriate to a given situation. Wu-wei is said to be the attitude of Taoist saint. In the Tao-teaching, Lao-tzu describes wu-wei as follows, "In pursuit of learning, everyday something is acquired (as regards our conceptual knowledge.). In pursuit of the Tao, everyday nothing is left undone. Wu-wei therefore does not denote absolute non-action but rather a form of action that is free of desire, intention, or motivation" (Kang, 2004)[10].

Central to the therapist's development of wu-wei is the requirement that she stops the habit of "projection" in therapy and, by this, "purify" her mind. It is important to note that the meaning of "projection" in Tao psychotherapy has a somewhat different emphasis than it does in Psychoanalysis. In Tao psychotherapy projection quite often refers to the therapist's act of imposing interpretations on clients as well as the client's acts of imposing interpretations onto their lives. According to Rhee (1993, 1995)[5][6], "projection" is one of basic mechanisms of human suffering and mental disturbance. The relationship between the unconscious and its mechanism of projection is clearly presented by Lee (2003)[11] who has been practicing and studying Tao therapy for several years and also the past president of Korean Academy of Psychotherapists (KAP). He provides a model of the process and the mechanism of projection of the unconsciousness and consciousness (Lee, 2003)[11].

According to Lee(2003)[11] and Eun (2004)[12], Won Hyo, who was the master of Buddhistic teaching distinguished three components to the unconsciousness: the activating mind (up-shik), the evolving mind (chun-shik) and the reproducing mind (hyun-shik). The activating mind is the initial moving mind which is reservoir of past experience both positive and negative. The activating mind is turned into the evolving mind. Evolving mind can be explained as one able to freely see the image which was evoked by activating mind. This evolving mind is turned into the reproducing mind. Reproducing mind is as if there is no boundary between you and 
the thing which is on the outside, like a mirror reflects the image of things. Then in consciousness, we have the analytic mind (ji-shik) like a knowledge which is followed by the continuing mind (sang-sok-shik). The continuing mind is a series of delusions. These two are the product of "projection". Philosophy and knowledge are all their derivatives. If the evolving, reproducing, analytical and continuing minds are purified through in Buddhistic practice, "working through (hoon-seup)" and only the residual that is the activating mind remains unpurified, it is Bodhisattva. When even this residual completely purifies, it is called Buddha. Buddha means the State of "no projection, or no unconsciousness, but always be awakened, and no thought". The essence of the teaching of Buddhism is "Stop and see," i.e. "Do not take the outside appearances, and illuminate your own mind." That is, it means correcting cognitive distortion through a purification of mind. In Confucianism, it is said that the right way to the Tao is to seek within oneself, not outside oneself.

\section{Method}

The biographical narrative method is often recommended for cultural studies (Megias, et al, 2017)[1][2] This approach seeks epistemological alternation, ideologically shattering the traditional division of so-called dominant scientific approach. Megias et al. (2017)[1] state as follows, "Biographical narrative approach strive to reevaluate key concepts such as knowledge, objectivity, subject/object relationship, and the nature of the cognoscente subject, which previously been unquestionable (p.973)". According to Bruner (1987, 1988)[13][14], a way of constructing, based on being, in which subjectivity is a condition necessary for social understanding. Personal experience is communicated as a story and the combined reflection of the subject's specific reconstruction of the experience give meaning to what was lived the events in question (Ricoeur, 1995)[15]. Therefore, question to explore similarities between Tao psychotherapy and Person-centered psychotherapy can be explained by a subject who has experience in both fields extensively.

\subsection{Sample}

\subsubsection{Narrative of Person-centered Psychotherapy Experience}

Currently, a middle-aged female full-time faculty member at a university, was born in Korea, but spent her childhood and adolescent life in the United States. In 1990, she started Ph.D. 
studies at the Mental Health Ph.D. Program in the department of psychology at the University of Chicago. The psychotherapist was trained at the Chicago Counseling and Psychotherapy Center established by Carl Rogers which carries Person-Centered spirit. The earlier version of the CCPC was student guidance center at the University of Chicago and later the center became a collaborative place where several professionals who practice by Person-Centered approach continues to carry the spirit of Carl Rogers's work. At the center, therapists would study articles, books written by Carl Rogers, examine his cases by analyzing distinguished Person-Centered orientation professionals offered videotapes and workshops, as well as many presentations. The psychotherapist's first impression of Rogers was that he is quiet, calm and sincere in his work. The tapes that she watched such as cases of Miss Munn, Gloria, etc. didn't appear to be like therapy, but rather like a having a serious conversation with a person. The psychotherapist started to learn and practice Person-Centered psychotherapy by entering the center's practicum and internship program which includes countless hours of supervision and practice.

The most striking experience she had when she was learning Person-Centered psychotherapy were the characteristics of warmth and humanity. The psychotherapist was deeply impressed by how devoted CCPC members were to teaching and practicing the principles of Person-Centered psychotherapy in both their personal and professional lives. They accepted her unconditionally and as a foreign student that acceptance mattered a great deal to her. The members at the center sincerely sought to develop egalitarian, democratic relationships, not only with clients, but also with each other. For example, whoever happened to come in first in the morning would clean and organize the counseling rooms and she doesn't remember any authoritative expectation from any of my supervisors to be the one to do so, even though she was just an intern therapist. Also many of the supervision sessions were used for personal therapy as the need arose to improve her work. The psychotherapist soon realized that in order to understand her clients, she had to open up and understand herself more deeply and accurately in order to be helpful and effective with my clients.

\subsubsection{Narrative of Tao Psychotherapy Experience}

In 1993, the psychotherapist visited Korea in order to collect data for Ph.D. dissertation. She was working on a project called "International Study of the development of psychotherapist", a project which aims to examine the nature, correlates, and influences in the development of professional psychotherapists from many cultures, countries, theoretical orientations and career levels (Orlinsky et al., 1999)[16]. As it is often the case, it was challenging to collect data 
concerning therapists and the therapist had difficult time getting questionnaires returned. Though she contacted numerous associations and academies, it was the members of the Korean Association of Psychotherapists (KAP) who were most enthusiastic and cooperative about the project. It seemed that KAP members appreciated the importance of both their personal and professional development as well as open to new learning. That is how the psychotherapist made affinity with KAP and Dr. Rhee Dongshick and was impressed by their rigorous training and strict supervision hours. To her, Dr. Rhee seemed so energetic and witty. He made her feel very comfortable though she was tense meeting him for the first time. Though at first, the atmosphere of academy appeared to be hierarchical and formal, hardly the egalitarian atmosphere the psychotherapist found at the University of Chicago, she was soon able to adjust and actively participated in many of the conferences and workshops, soon becoming a member of the board of trustees and worked for KAP for many years afterward.

\subsection{Analysis}

The Biographical narrative interpretive method implies a thorough process leading towards an understanding of the individual case by so-called "narrative turn" (Clandinin \& Connelly, 1994)[17]. This means that according to Megias et al. (2017)[1], "people are no longer considered as research objects/subjects. Instead, they are conceptualized as biographic individual capable of action who possess knowledge-building assets and different outlooks on the worlds (p. 963). In this context, the psychotherapist who is the subject as well as researcher tells and

organizes her expertise in both Tao psychotherapy and person-centered experience. Her recollections, memos, journal notes, research articles, etc. will be used.

\section{Results}

\subsection{Similarities}

The similar points between Tao psychotherapy and person-centered therapy are mostly related to six necessary and sufficient conditions need to provide in person-centered orientation which are therapist's genuineness, unconditional positive regard and empathic understanding. The similarities can be summarized as follows.

The first point is the need for a therapist's maturing his/her personality, purification of mind. This aspect is important in both Tao therapy and the Person-Centered approach. The 
Person-Centered approach emphasizes therapists' genuineness, acceptance, self-awareness, etc., which are all the qualities for a mature personality so that he or she can provide therapeutic environment for clients. The significant relationship between the extent of constructive personality change in the client and four counselor variables: (a) the degree of empathic understanding of the client manifested by the counselor; (b) the degree of positive affective attitude (unconditional positive regard) manifested by the counselor toward the client; (c) the extent to which the counselor is genuine, his words matching his own internal feelings; and (d) the extent to which the counselor's response matches the client's expression in the intensity of affective expression (Kirschenbaum \& Henderson, 1989)[18]. Therefore, in Person-Centered therapy, a therapist's maturing personality is a tool or a manner in order to achieve the goal. In Tao therapy, the level of the therapist's maturing personality is higher, up to the level of "purification of mind". The therapist becomes in a Zen state and so transparent, in other words, transcend-self and this is not a tool to achieve something. According to Lee (1990)[19], the aim of Western psychotherapy lies in the dimension of self-understanding, while the aim of Eastern approach lies in self-transcendence. In Tao therapy, therapists need to be in self-transcendence state, and this can be naturally therapeutic because optimal empathy can be attained when a therapist is in this being. This is equivalent to Person-centered approach that is when therapist's words are in line with his feelings rather than the two being discrepant. Also, it will be effective if the counselor likes the client unconditionally and if the counselor understands the essential feelings of the client then there is a strong probability.

The second point is that the therapist's presence itself can be therapeutic. When a client is in a therapy room with a Tao therapist who is fluently in wu-wei, the client is able to experience his or her true self. In the Person-Centered approach, clients are encouraged to express his or her internal feelings, in other words, open to experience. In Tao therapy, there is no need for a client to be verbal and express what is happening inside, but by just being with that therapist, the client can be freed from all external conditions. This fits well with the expectation of Korean clients that they expect the therapists to be in the authoritative role. For example, in a Tao psychotherapy case conducted by Dr. Rhee, a client reported that he dreamed of two suns rising into the sky, and he knew one of them was his father and the other was the Dr. Rhee. Before entering the therapy, the client did not know that his problems were linked to his father. The client expressed that he was glad he experienced that dream and was able to resolve unfinished issues with his father (Kim \& Rim, 2003)[20]. 


\subsection{Differences}

The different points between Tao psychotherapy and person-centered therapy lie mostly related to cultural differences in which the two orientations are based on. Tao psychotherapy based on Eastern culture emphasizes contextual whereas person-centered orientation based on Western culture stress individualized existence. The differences can be summarized as follows.

The first point is that therapists need to deal "self-in-relations". In Tao therapy, empathy naturally comes from compassion or Zen mind. The therapist enters into a patient's mind to the extent that the subject-object congruence will be established. Aside from the client, the therapist is able to empathize with the client's parents, friends, and other people in his or her life. This approach is extremely important in the collectivistic culture of a non-Western setting. The collectivistic characteristic of non-Western cultures and individualistic characteristics of Western have often been differentiated on the basis of the notion of independent and interdependent self-construal (Markus \& Kitayama, 1991, 1994, 1998; Singelis, 1994)[21-24]. The type of cultural orientation (independent versus interdependent) in which individuals are socialized has a strong impact on their behaviors, thoughts, emotions, and motivations.

People with interdependent self-construal are attentive, connected, and responsive to others (Kondo, 1990)[25]. Those with interdependent ways of being find a way to fit in with others who are close to them, to become inextricably linked to various interpersonal relationships and to fulfill role obligations (Miller, 1988; Shweder \& Bourne, 1984)[26][27]. The ability to control behaviors, thoughts, emotions, and motivations to accommodate others is an important contribution to the self-esteem of interdependent selves because self-restraint is required to put others' needs and desire first (Markus \& Kitayama, 1991)[21]. Therefore, seeing clients in a non-Western culture such as Korea, the therapist needs to consider the client not as an independent self, but as an interdependent self. For example, if a 30-year-old depressed woman came into therapy expressing how frustrating it is to experience social and family pressure to get married, the Tao therapist might say, "You must be very distressed by the pressure, but also it must be difficult for your mother to have an unmarried daughter of your age." Here, the therapist is empathizing with both the client and her significant person, her mother. In this way, the client can experience feeling understood and at the same time be able to see her mother's point of view, which is a significant influential factor on who the client is.

The second point it that no one is centered upon therapy and the therapy is holistic. In Tao therapy, no one is centered. It is not about client per se, or therapist per se. In Tao therapy both therapist and client change and develop. The notion of the Tao is the origin of all 
creation and the force which is unknowable in its essence but observable in its manifestation that lies behind the functioning and changes of the natural world. Based on this foundation, in Tao therapy, no one is centered upon in the therapy. That is it is not client-centered, therapist-centered, or person-centered. It expands to not only living but non-living things. The philosophy behind Tao therapy is that everything in this universe is intertwined and connected so it is not possible to emphasize a certain organism over any other organism. This kind of holistic approach enables the therapist to be more humble and helps the client to place his or her problems within a broader perspective.

The third point is that therapy can be brief and directive. Person-centered therapy is a non-directive approach, i.e., the therapist does not guide or direct the client. However, Tao therapy can be directive. When the therapist is in wu-wei, he or she can reach optimal empathy with the client and to directly point to the client's core problem. This approach applies well with the client's expectations of counseling or psychotherapy within Korea. Culturally, in Korea, therapists are expected by most clients to play a role akin to teachers. The teacher-student or senior-junior (sun-bae/ who-bae) relationship is strongly hierarchical in the sense that students are trained to obey their teachers and expect teachers to guide and lead them (Joo, 1996)[28]. However, it is important to point out that a directive approach is not done in a negative way. According to Rodin (1997)[29], who examined several Tao therapy sessions, the clinical materials demonstrate a "seamless" interview in which the therapist is in tune with the client and provides an integrative function done in a direct manner. In other words, the therapist expresses empathy in a direct and immediate way at the same time being very easy and gentle with a client. Since Tao therapy does not involve specific techniques, it is not easy to understand. Due to the lack of initial experience with psychotherapy and generally poor mental health system, Korean clients expect therapy to be brief. This tendency is prevalent among clients with Asian cultural background. Therefore, it is not at all uncommon for Tao psychotherapy to be as brief as two or three sessions. According to Kim, Atkinson \& Umemoto (2001)[30], clients of Asian origin have great respect for counselors and therapists, but also expect to be provided with definite answers and direct guidance in dealing with problems in a short period of time. Therefore, when this expectation is not met, there is a high drop out rate among clients. In Tao therapy, because the therapist is able to detect the core problem of clients, a client does not have to wait long for results. The therapist will deliver the core issues of the client, and the client, who has high respect for the therapist, is ready to accept it and will enter the process of change.

Examining the similarities and differences between Person-centered and Tao psychotherapy, 
similarities are about therapist themselves, their personality and being. It seems that psychotherapeutic culture is greater than national culture (Bae, Joo \& Orlinsky, 2003)[31]. In terms of differences, they were mostly about approaches that therapists need to be aware of clients' national cultural background. It has been constantly discussed that modern theories of psychotherapy imported from the West may not give Korean therapists sufficient guidance for the complex cultural situation in which they practice (Kang, 1996; Rhee, 1995)[11][6]. Since Tao psychotherapy was developed in the context of Korean culture, Tao therapists emphasize clients' self-in-relation and provide holistic, brief and directive approach.

\section{Conclusion}

In sum, when a Tao therapist is in wu-wei, he or she can reach optimal empathy with client. This state may be similar to what Rogers calls therapist's 'congruence'. Empathy naturally comes from this kind of Zen mind or compassion. It can be said that the therapist enters into client's mind to the extent that the subject-object congruence will be established. The therapist is also able to empathize not only with the client, but with his or her parents, friends, or other people related to his or her life. In the Tao, everything is connected in relation, i,e, no separate individual entity exists. This may be unique to Tao therapy which is based on Eastern culture which is contextual, circular and holistic. At this stage, the therapist is able to detect a client's subjective "Nuclear Feeling". This is similar to what Rogers calls 'empathic understanding'. It is the core, essential or foundation of a client's distress or a problem that holds sway over the patient's mind through every moment of the patient's behavior. By therapeutic communication in an empathic and mostly brief and non-verbal way, the therapist helps the client to recognize, control, and resolve his or her own "Nuclear Feelings". A Tao therapist does this by "directly pointing at the Nuclear Feelings". This is similar to teachings in Buddhism that is called "finger pointing at the moon". In this case, moon means reality and a client's subjective feelings. "Finger" means words, concepts, and theory. In Tao therapy, when you have seen the "moon", you should forget the "finger". When you are attached to the conceptual theory, the reality becomes distorted. That is why Tao is not a theory, but it is a way of looking at things as they really are. In other words, a Tao therapist is able to empathize with client's subjective experience directly.

So how do the psychotherapist bridge Tao therapy and person-centered therapy in practice? As the meaning of Tao suggests, the way of doing things naturally, she just try to be herself and practice therapy. Born and raised in an Eastern country, Korea as well as many years of 
staying in a Western country, America, she naturally developed both Eastern part and Western aspects of her personality. This is helpful since Korea is rapidly Westernized and modernized, many of clients have different expectation about therapy and issues compared to clients from ten or twenty years ago (Joo, 2013)[32]. Although, contemporary Koreans still hold traditional beliefs and attitudes with respect to accepting group obligations, during the last three decades, Korean has changed from $\mathrm{s}$ traditional agrarian society to a predominantly young, urban, educated industrial society with remarkable economic growth (Hyun, 2001)[33]. As a result, currently Koreans are relatively more individualistic than in previous generation and this is especially true for younger Koreans. Therefore, not Tao therapy or person-centered orientation alone will work for Korean clients. This fits very well with results from Wampold et al (1997)[34]'s Meta-analysis study that no single therapeutic theory exists which is effective for all clients. They humorously explains this by quoting a line from Alice in Wonderland about Dodo bird's judgment, "Everybody has won, and all must have prizes" (Caroll, 1865/1962, p.412)[35]. So, the psychotherapist's of bridging Tao therapy and person-centered approach continues.

\section{Acknowledgement}

This research was supported by Duksung Women's University Research Grants 2019.

\section{References}

[1] Megias, M. E., Garcia, M, J., Arcos, D., Life stories as biographic-narrative method, How to listen to silenced voices, Procedia- Social and Behavioral Sciences, (2017), Vol.237, pp.962-967. DOI: 10.1016/j.sbspro.2017.02.136

[2] Ramvi, E., I am only a nurse: a biographical narrative study of a nurse's self-understanding and its implication for practice, BMC Nursing, (2015), Vol.14, No.23, pp.1-9. DOI: 10.1186/s12912-015-0073-y

[3] Joo, E., Psychotherapy in South Korea: A Preliminary Exploration of Past, Present and Future Challenges, Korean Journal of Psychology: General (Korean J Psychol Gen), (2006), Vol.25, No.2, pp.103-120. UCI : G704-001037.2006.25.2.001

[4] Craig, E., Tao psychotherapy: Introducing a New Approach to Humanistic practice, The Humanistic Psychologist, (2007), Vol.35, No.2, pp.109-133. DOI:10.1080/08873260701274074

[5] Rhee, D., The Tao and empathy: East Asian interpretation, Journal of Korean Academy of Psychotherapists, (1993), Vol.7, No.1, pp.1-13. 
[6] Rhee, D., The Tao and Western psychotherapy, Psychotherapy East and West, Proceedings of the 16th International Congress and Psychotherapy, (1995), August 21-25; Seoul, Korea.

[7] Rogers, C. R., On becoming a person: A therapist's view of psychotherapy, Houghton Mifflin Company, (1961)

[8] Brynner, W., The way of life according to Lao Tzu, Perigree Books, (1986)

[9] Lao Tzu, Tao Te Ching, Shambala, (1961)

[10] Kang, S. H., Ways to be a psychotherapist and a bodhisttva, International Forum on Taopsychotherapy and Western Psychotherapy, (2004), August 21-22; Seoul, Korea.

[11] Lee, J. K., What is Tao-Psychotherapy?, Conference of Asia-Pacific Association of Psychotherapists, (2003), August 6-9; Singapore.

[12] Eun, J. H., TWon Hyo's Dae seung ki shin lon, Il-bup-sa, (2004)

[13] Bruner, J., Life as narrative, Social Research, (1987), Vol.54, No.1, pp.11-32.

[14] Bruner, J., Desarrollo cognitivo y educacion, Ediciones Marata, S. L. (1988)

[15] Ricoeur, P., Tiempo y narracion 1, Configuracion del tiempo en el relato historico, Siglo XXI, (1995)

[16] Orlinky, D. E., Ambuhl, H., Willutzki, U., Dazord, A., Ronnestand, M. H., Davis, J. D., Gerin, P., Davis, M., Botermans, J. F., Cierpka, M., The development of psychotherapists: Concepts, questions, and methods of a collaborative international study, Psychotherapy Research, (1999), Vol.9, No.2, pp.127-153. DOI:10.1093/ptr/9.2.127

[17] Clandinin, D. J., Connelly, F. M., Personal Experience Methods. In Denzin, N.K.,\&Y.S.Lincoln(eds.), Handbook of Qualitative Research, Thousand Oaks, CA: Sage, (1994)

[18] Rogers, C. R., Kirschenbaum, H., Henderson, V. L., The Carl Rogers reader, Boston: Houghton Mifflin Company, (1989)

[19] Lee, C. H., A comparison of Oriental and Western approach to counseling and guidance, Korean Journal of Counseling and Psychotherapy, (1990), Vo1.3, No.3, pp.1-8.

[20] Kim, J. H., Rim, H. D., Initial interview and diagnosis of the nuclear feelings, Conference of Asia-Pacific Association of Psychotherapists, (2003), August 6-9; Singapore.

[21] Markus, H. R., Kitayama, S. D., Culture and the self: Implications for cognition, emotion, and motivation, Psychological Review, (1991), Vol.98, No.2, pp.224-253. DOI: 10.1037/0033-295X.98.2.224

[22] Markus, H. R., Kitayama, S., A collective fear for the collective: Implications for selves and theories of selves, Personality and Social Psychology Bulletin, (1994), Vol.20, No.5, pp.568-579. DOI: $10.1177 / 0146167294205013$

[23] Markus, H. R., Kitayama, S., The cultural psychology of personality, Journal of Cross-Cultural Psychology, (1998), Vol.29, No.1, pp.63-87. DOI: 10.1177/0022022198291004

[24] Singelis, T. M., The measurement of independent and interdependent self-construals, Personality and Social Psychology Bulletin, (1994), Vol.20, No.5, pp.580-591. DOI: 10.1177/0146167294205014 
[25] Kondo, D. K., Crafting Selves: Power, Gender, and Discourses of Identity in a Japanese Workplace, University of Chicago Press, (1990)

[26] Miller, J. G., Bridging the content-structure dichotomy: Culture and the self, In M.H. Bond (Ed.), The cross-cultural challenges to social psychology, Sage, (1988)

[27] Shweder, R. A., Bournes, E. J., Does the concept of the person vary cross-culturally? In R.A. Shweder \& R.A. Levine (Eds.), Culture theory: Essays on mind, self, and emotion, England: Cambridge University Press, (1984), pp.158-199.

[28] Joo, E., The psychotherapeutic relationship in Korea : cross-national and internal cultural comparison, University of Chicago, Unpublished doctoral dissertation, (1997)

[29] Rodin, G., T. Reactions to Dr. Dongshick Rhee's interview, Psychotherapy Workshop, (1997), November 30; Seoul, Korea.

[30] Kim, B. S. K., Atkinson, D. R., Umemoto, D., Asian cultural values and the counseling process: Current knowledge and directions for future research, The Counseling Psychologist, (2001), Vol.29 No.4, pp.570-603. DOI: $10.1177 / 0011000001294006$

[31] Bae, S. H., Joo, E., Orlinsky, D. E., Psychotherapists in South Korea: Professional and Practice Characteristics, Psychotherapy: Theory, Research, Practice, Training, (2003), Vol.40, No.4, pp.302-316. DOI: 10.1037/0033-3204.40.4.302

[32] Joo, E., Counseling and psychotherapy in South Korea: Disciplines flourishing in a dynamic and challenging era, Handbook of Counseling and Psychotherapy in an International Context, Routledge, (2013)

[33] Hyun, K. J., Sociocultural changes and traditional values: Confucian values among Koreans and Korean Americans, International Journal of Intercultural Relations, (2001), Vol.25, No.2, pp.203-229. DOI: $10.1016 / \mathrm{S} 0147-1767(01) 00009-8$

[34] Wampold, B. E., Mondin, G. W., Moody, M., Stich, F., Benson, K., Ahn, H., A meta-analysis of outcome studies comparing bona fide psychotherapies: Empirically "all must have prizes.", Psychological Bulletin, (1997), Vol.122, No.3, pp.203-215. DOI: 10.1037/0033-2909.122.3.203

[35] L. Caroll, Alice's adventures in wonderland, Hamondsworth, Middlesex, England, Penguin Books, (1962) 